H. K. Kim

Nagoya Math. J.

Vol. 112 (1988), 117-124

\title{
UNIT THEOREMS ON ALGEBRAIC TORI
}

\author{
HYUN KWANG KIM
}

Let $k$ be a $p$-adic field (a finite extension of $\boldsymbol{Q}_{p}$ ) or an algebraic number field (a finite extension of $\boldsymbol{Q}$ ). Let $T$ be an algebraic torus defined over $k$. We denote by $\hat{T}$ the character module of $T$ (i.e. $\hat{T}=$ $\left.\operatorname{Hom}\left(T, G_{m}\right)\right)$, where $G_{m}$ is the multiplicative group.

As is well-known (cf. [7]), $T$ is split by a finite galois extension $K / k$. We denote by $G$ the galois group of $K / k$. Then $\hat{T}$ becomes naturally a $G$-module. Since the map $T \rightarrow \hat{T}$ yields a canonical isomorphism between the category of tori defined over $k$ and split by $K$ and the dual category of finitely generated $Z$-free $G$-modules, it is natural to use $\operatorname{Hom}_{\theta}\left(\hat{T}, M_{K}\right)$ as a definition of an object relative to $T$ over $k$ when $M_{K}$ is a $G$-module of arithmetical interest related to $K$.

In this paper, we will determine the structure of $\operatorname{Hom}_{\theta}\left(\hat{T}, O_{K}^{\times}\right)$where $O_{K}^{\times}$is the group of units of $K$ and will discuss the meaning of this group.

\section{§1. Local unit theorem}

Let $k$ be a $p$-adic field. First we recall the structure of $O_{k}^{\times}$. Let $\pi$ be a prime element of $k$ and let $U_{1}$ be the group of one units of $k$ i.e. $U_{1}=1+\pi O_{k} . \quad Z_{p}$ acts on $U_{1}$ as follows:

Let $a=a_{0}+a_{1} p+\cdots+a_{n} p^{n}+\cdots \in Z_{p}$ and $u \in U_{1}$. Set $a_{n}=$ $\sum_{i=0}^{n} a_{i} p^{i}$. Then $\left\{u^{a_{n}}\right\}$ is a Cauchy sequence in $U_{1}$. Since $U_{1}$ is compact, the limit exists and denoted by $u^{a}$.

So we can view $U_{1}$ as $Z_{p}$-module. We have the following proposition (cf. [5]).

(1.1) Proposition. $U_{1} \approx W\left(U_{1}\right) \times Z_{p}^{\left[k . Q_{p}\right]}$, where $W\left(U_{1}\right)$ is the group of roots of unity in $U_{1}$.

Now $O_{k} /(\pi)$ has $q=p^{s}$ elements. Let $\eta$ be a primitive $(q-1)$ th root of unity in $O_{k}$. Then

Received April 27, 1987. 


$$
O_{k}^{\times}=\langle\eta\rangle \times U_{1} \approx\langle\eta\rangle \times W\left(U_{1}\right) \times Z_{p}^{\left[k: Q_{p}\right]} .
$$

We have proved

(1.2) Proposition. Let $k$ be a p-adic field. Up to finite torsions, $O_{k}^{\times}$is a free $Z_{p}$-module of rank $\left[k: \boldsymbol{Q}_{p}\right]$.

Let $k$ be a $p$-adic field and $T$ be a torus defined over $k$ split by $K$, where $K$ is a finite galois extension of $k$ with galois group $G$. We can think $\operatorname{Hom}\left(\hat{T}, O_{K}^{\times}\right)$as a $G$-module. Let $\operatorname{Hom}_{G}\left(\hat{T}, O_{K}^{\times}\right)$denote the $G$ invariant submodule of this module.

(1.3) Definition. $T\left(O_{k}\right)=\operatorname{Hom}_{G}\left(\hat{T}, O_{K}^{\times}\right)$

We have the following main theorem for local theory.

(1.4) Theorem. Up to finite torsions, $T\left(O_{k}\right)$ is a free $Z_{p}$-module of $\operatorname{rank} r(T)=\left[k: \boldsymbol{Q}_{p}\right] \cdot(\operatorname{dim} T)$.

Proof. By Proposition 1.2,

$$
O_{K}^{\times}=W \times U_{1} \text {, where } W \text { is a finite group. }
$$

Therefore,

$$
T\left(O_{k}\right)=\operatorname{Hom}_{G}(\hat{T}, W) \times \operatorname{Hom}_{G}\left(\hat{T}, U_{1}\right) .
$$

Since $\operatorname{Hom}_{G}(\hat{T}, W)$ is a finite group, it suffices to determine the $Z_{p}$-module structure of $\operatorname{Hom}_{G}\left(\hat{T}, U_{1}\right)$. For each $m \geq 1$, set $U_{m}=1+\left\langle\pi^{m}\right\rangle$.

It is well-known that (cf. [5]):

(i) $U_{m}$ is a $Z_{p}$-submodule of $U_{1}$ of finite index.

(ii) $U_{m}$ is free if $m>\frac{e}{p-1}$, where $e$ is the ramification index of $p$ over $K$.

We will determine the $Z_{p}$-rank of $\operatorname{Hom}_{G}\left(\hat{T}, U_{m}\right)$ for sufficiently large $m$.

Now we need lemmas.

(1.5) Lemma. Let $R$ be a commutative ring and $M, N$ be $R$-modules. We have an isomorphism

$$
\operatorname{Hom}_{R}(M, N) \approx M^{*} \otimes_{R} N,
$$

where $M^{*}=\operatorname{Hom}_{R}(M, R)$ denote the dual module of $M$. Assume further that a finite group $G$ acts on $M$ and $N$. Then the isomorphism induces an isomorphism of $G$-invariant parts. 


$$
\operatorname{Hom}_{R[G]}(M, N) \approx\left(M^{*} \otimes_{R} N\right)^{G}
$$

Proof. See Proposition 10.30 in [2].

(1.6) Lemma. Let $R$ be a principal ideal domain and let $K$ be its quotient field. Let $X$ be a finitely generated $R$-free module. Assume that a group $G$ acts on $X$. Then

$$
\operatorname{rank}_{R} X^{G}=\operatorname{dim}_{K}\left(X \otimes_{R} K\right)^{G} .
$$

Proof. It sufficies to show $X^{G} \otimes_{R} K=\left(X \otimes_{R} K\right)^{G}$. Clearly $X^{G} \otimes_{R} K \subset$ $\left(X \otimes_{R} K\right)^{G}$. To do converse, choose a basis $\left\{x_{1}, \cdots, x_{n}\right\}$ of $X$ over $R$ such that $\left\{a_{1} x_{1}, \cdots, a_{l} x_{l}\right\}$ is a basis of $X^{G}, a_{1}, \cdots, a_{l} \in R$. Assume $x=x_{1} k_{1}+$ $\cdots+x_{n} k_{n}, k_{i} \in K$, be an element of $\left(X \otimes_{R} K\right)^{a}$. We can choose $r \in R$ such that $k_{i} r \in R$ for all $i=1, \cdots, n$. Hence $x r=x_{1} k_{1} r+\cdots x_{n} k_{n} r \in X^{G}$. By the choice of our basis, we have $k_{i} r=0$ if $i>l$. This proves that $x \in X^{G} \otimes_{R} K$.

(1.7) Lemma. Let $V$ be a vector space over a field $K$, char $K=0$. Let $\varphi: G \rightarrow G L(V)$ be a representation of $G$ in $V$. Then

$$
\operatorname{dim}_{K} V^{G}=\frac{1}{|G|} \sum_{g \in G} \chi(g)
$$

where $\chi$ is the character of $\varphi$.

Proof. First assume that $\varphi$ is irreducible. Then $V^{G}=0$ or $G$.

(i) $V^{G}=V$. Then $\varphi(g)=\mathrm{id}_{V}$ for all $g \in G$.

Hence

$$
\frac{1}{|G|} \sum_{g \in G} \chi(g)=\frac{1}{|G|} \sum_{g \in G}(\operatorname{dim} V)=\operatorname{dim} V .
$$

(ii) $V^{G}=0$.

Let $\left\{v_{1}, \cdots, v_{n}\right\}$ be a basis of $V$ over $K$ and let $\left(a_{i j}(g)\right)$ be the matrix of $\varphi(g)$ with respect to this basis. For each $i$,

$$
\sum_{g \in G} \varphi(g) v_{i} \in V^{G}=0 .
$$

On the other hand,

$$
\sum_{g \in G} \varphi(g) v_{i}=\sum_{g \in G}\left(\sum_{j} a_{i j}(g) v_{j}\right)=\sum_{j}\left(\sum_{g \in G} a_{j i}(g)\right) v_{j} .
$$

By linearly independence, 


$$
\sum_{g \in G} a_{j i}(g)=0 \quad \text { for all } i, j=1, \cdots, n
$$

Hence

$$
\sum_{g \in G} \chi(g)=\sum_{g \in G}\left(\sum_{i} a_{i i}(g)\right)=\sum_{i}\left(\sum_{g \in G} a_{i i}(g)\right)=0 .
$$

For general case, let $V=V_{1} \oplus \cdots \oplus V_{k}$ be a decomposition of $V$ into irreducible subspaces. So we have $V^{G}=V_{1}^{G} \oplus \cdots \oplus V_{k}^{G}$. Let $\chi_{i}$ be the character of the subrepresentation $\varphi_{i}: G \rightarrow G L\left(V_{i}\right)$. By the first case,

$$
\operatorname{dim} V_{i}^{G}=\frac{1}{|G|} \sum_{g \in G} \chi_{i}(g) .
$$

Hence

$$
\operatorname{dim} V^{G}=\sum_{i} \operatorname{dim} V_{i}^{G}=\sum_{i}\left(\frac{1}{|G|} \sum_{g \in G} \chi_{i}(g)\right)=\frac{1}{|G|} \sum_{g \in G} \chi(g) .
$$

To apply Lemma 1.5 to our problem we need:

Sublemma. There is a natural isomorphism

$$
\operatorname{Hom}_{Z}\left(\hat{T}, U_{m}\right) \approx \operatorname{Hom}_{Z_{p}}\left(\hat{T} \otimes Z_{p}, U_{m}\right) .
$$

Furthermore,

$$
\operatorname{Hom}_{Z[G]}\left(\hat{T}, U_{m}\right) \approx \operatorname{Hom}_{Z_{p[G]}}\left(\hat{T} \otimes Z_{p}, U_{m}\right) .
$$

Proof. Straightforward.

By abuse of notation, we will write $\hat{T}$ instead of $\hat{T} \otimes Z_{p}$. Assume that $m>\frac{e}{p-1}$. Then $U_{m}$ is $Z_{p}$-free.

By Lemma 1.5,

$$
\operatorname{Hom}_{G}\left(\hat{T}, U_{m}\right)=\left(\hat{T}^{*} \otimes U_{m}\right)^{G}
$$

By Lemma 1.6,

$$
r(T)=\operatorname{rank}_{Z_{p}}\left(\hat{T}^{*} \otimes U_{m}\right)^{G}=\operatorname{dim}_{Q_{p}}\left(\hat{T}^{*} \otimes U_{m}\right)^{G} .
$$

Assume that $G$ acts on $\hat{T}$ and $U_{m}$ with characters $\chi_{1}$ and $\chi_{2}$, respectively. Let $\chi$ be the character comes from the action of $G$ on $\hat{T}^{*} \otimes U_{m}$. Then

$$
\chi(\sigma)=\chi_{1}\left(\sigma^{-1}\right) \cdot \chi_{2}(\sigma) \quad \text { for all } \sigma \in G
$$

By Lemma 1.7, 


$$
r(T)=\frac{1}{|G|} \sum_{\sigma \in G} \chi_{1}\left(\sigma^{-1}\right) \cdot \chi_{2}(\sigma)=\left\langle\chi_{1}, \chi_{2}\right\rangle .
$$

Now we will describe the action of $G$ on $U_{m}$.

Sublemma. Let $|G|=n$. There exists $\pi^{\prime}$ in $\pi^{n} O_{k}$ such that $\sigma\left(\pi^{\prime}\right)=\pi^{\prime}$ for all $\sigma \in G$.

Proof. Put $\pi^{\prime}=\prod_{\sigma \in G} \sigma(\pi)$.

Assume that $m>\frac{e}{p-1}$ and $|G|=n / m$. By the above sublemma, we may assume that $\sigma\left(\pi^{m}\right)=\pi^{m}$ for all $\sigma \in G$. We have the following commutative diagram:

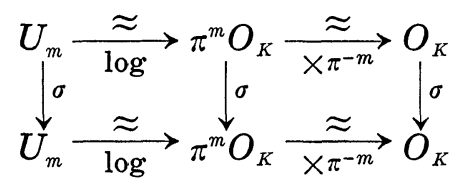

Choose a normal basis $\left\{x^{\sigma}\right\}_{\sigma \in G}$ of $K$ over $k$, and let $\left\{\alpha_{1}, \cdots, \alpha_{m}\right\}$ be a basis of $k$ over $\boldsymbol{Q}_{p}$. Then $\left\{\alpha_{i} x^{\sigma}\right\}_{\substack{i=1, \ldots, m \\ \sigma \in G}}$ forms a basis of $K$ over $\boldsymbol{Q}_{p}$. By multiplying some power of $\pi$ which is invariant under the action of $G$, we may assume that $\alpha_{i} x^{\sigma} \in O_{K}$ for all $\sigma \in G$ and $i=1, \cdots, m$. By the above diagram $\left\{\exp \left(\pi^{m} \alpha_{i} x^{\sigma}\right)\right\}_{\substack{i=1, \cdots, m \\ \sigma \in G}}$ forms a basis of $U_{m}$ over $Z_{p}$. So we have

$$
\chi_{2}(\sigma)= \begin{cases}m \cdot|G| & \text { if } \sigma=\text { identity } \\ 0 & \text { otherwise. }\end{cases}
$$

Therefore

$$
\begin{aligned}
r(T) & =\frac{1}{|G|} \sum_{\sigma \in G} \chi_{1}\left(\sigma^{-1}\right) \chi_{2}(\sigma)=\frac{1}{|G|} \chi_{1}(\mathrm{id}) \cdot m|G| \\
& =m \cdot(\operatorname{dim} T)=\left[k: \boldsymbol{Q}_{p}\right] \cdot(\operatorname{dim} T) .
\end{aligned}
$$

(1.8) Remark. Take $T=G_{m}$ the multiplicative group. If we think $T$ is defined over $k$ and split by $k$, then Theorem 1.4 reduced to Proposition 1.2.

\section{§ 2. Global unit theorem}

Let $k$ be a number field, and $T, K, G$ be as in Section 1. As in Section 1, we define the $O_{k}$ point of $T$ as follows: 
(2.1) Definition. $T\left(O_{k}\right)=\operatorname{Hom}_{G}\left(\hat{T}, O_{K}^{\times}\right)$.

Then $T\left(O_{k}\right)$ becomes a $Z$-module. Let $r(T)$ denote its rank. By the arguments in Section 1, we have

$$
r(T)=\frac{1}{|G|} \sum_{\sigma \in G} \chi_{1}\left(\sigma^{-1}\right) \chi_{2}(\sigma)=\left\langle\chi_{1}, \chi_{2}\right\rangle,
$$

where $\chi_{1}$ is the character comes from the action of $G$ on $\hat{T}$ and $\chi_{2}$ is the character comes from the action of $G$ on $O_{K}^{\times}$.

Now we will describe the action of $G$ on $O_{K}^{\times}$. Let $m=[k: Q]$ and $n=[K: k]$. Let $k_{1}, \cdots, k_{\rho_{1}+\rho_{2}}, k_{\rho_{1}+\rho_{2}+1}^{\prime}, \cdots, k_{\rho_{1}+\rho_{2}+r_{2}}^{\prime}, k_{\rho_{1}+\rho_{2}+1}^{\prime \prime}, \cdots, k_{\rho_{1}+\rho_{2}+r_{2}}^{\prime \prime}$ be the distinct conjugates of $k\left(\rho_{1}+\rho_{2}+2 r_{2}=m\right)$. To each of them, we can correspond a conjugate of $K$ to which we will give the same index. The indices are chosen in the way that:

(i) For $1 \leq i \leq \rho_{1}, k_{i}$ and $K_{i}$ are real,

(ii) for $\rho_{1}<i \leq \rho_{1}+\rho_{2}, k_{i}$ is real and $K_{i}$ is imaginary,

(iii) for $\rho_{1}+\rho_{2}<i, k_{i}^{\prime}$ and $k_{i}^{\prime \prime}$ are complex conjugates and the same for $K_{i}^{\prime}$ and $K_{i}^{\prime \prime}$.

Note that $K_{i}$ is galois over $k_{i}$ whose galois group is isomorphic to $G$. So we may identify its galois group with $G$. Suppose that $\rho_{2} \neq 0$. Then $n$ is even. For $\rho_{1}<i \leq \rho_{1}+\rho_{2}, K_{i}$ is of degree 2 over the maximal real subfield of $K_{i} / k_{i}$. Let $H_{i}$ be the subgroup of $G$ corresponding to this field. We have the following proposition (cf. [3], [4]).

(2.2) Proposition. Let $H$ be the representation of $G$ on $O_{K}^{\times}, C$ be the trivial representation of $G, A$ be the regular representation of $G$ and $B_{i}$ be the induced representation of $G$ induced by the trivial representation of $H_{i}, \rho_{1}+1 \leq i \leq \rho_{1}+\rho_{2}$. Then we have

$$
H+C=\left(\rho_{1}+r_{2}\right) A+\sum_{i=\rho_{1}+1}^{\rho_{1}+\rho_{2}} B_{i} .
$$

Proposition 2.2 says that

$$
\chi_{2}=\left(\rho_{1}+r_{2}\right) \chi_{A}+\sum_{i=\rho_{1}+1}^{\rho_{1}+\rho_{2}} \chi_{B_{i}}-\chi_{C}
$$

Hence

$$
\left\langle\chi_{1}, \chi_{2}\right\rangle=\left(\rho_{1}+r_{2}\right)\left\langle\chi_{1}, \chi_{A}\right\rangle+\sum_{i=\rho_{1}+1}^{\rho_{1}+\rho_{2}}\left\langle\chi_{1}, \chi_{B_{i}}\right\rangle-\left\langle\chi_{1}, \chi_{C}\right\rangle
$$

On the other hand,

$$
\left\langle\chi_{1}, \chi_{A}\right\rangle=\frac{1}{|G|}(\operatorname{dim} T) \cdot|G|=\operatorname{dim} T
$$




$$
\begin{aligned}
\left\langle\chi_{1}, \chi_{C}\right\rangle & =\frac{1}{|G|} \sum_{\sigma \in G} \chi_{1}\left(\sigma^{-1}\right) \chi_{C}(\sigma)=\frac{1}{|G|} \sum_{\sigma \in G} \chi_{1}(\sigma)=\operatorname{rank} \hat{T}^{G} \quad \text { (by Lemma 1.7) } \\
\left\langle\chi_{1}, \chi_{B_{i}}\right\rangle & =\left\langle\left.\chi_{1}\right|_{H_{i}},\left.\chi_{B_{i}}\right|_{H_{i}}\right\rangle_{H_{i}} \text { (by Frobenius reciprocity law) } \\
& =\operatorname{rank} \hat{T}^{H_{i}} \quad \text { (by Lemma 1.7). }
\end{aligned}
$$

So we have proved

(2.3) Theorem. Let $T$ be a torus defined over a number field $k$. Up to finite torsions, $T\left(O_{k}\right)$ is a free Z-module of rank $r(T)$, where

$$
r(T)=\left(\rho_{1}+r_{2}\right) \cdot \operatorname{dim} T+\sum_{i=\rho_{1}+1}^{\rho_{1}+\rho_{2}} \operatorname{rank} \hat{T}^{H_{i}}-\operatorname{rank} \hat{T}^{G} .
$$

(2.4) Remark. T. Ono showed the following generalization of Dirichlet unit theorem (cf. [6]):

Let $T$ be a torus defined over $Q$. Then $Z$-rank of $T(Z)$ is $r_{\infty}-r_{Q}$, where $r_{\infty}=\operatorname{rank} \hat{T}(\boldsymbol{R})$ and $r_{\boldsymbol{Q}}=\operatorname{rank} \hat{T}(\boldsymbol{Q})$.

We can deduce this result from Theorem 2.3. Let $K$ be a splitting field of $T$ over $\boldsymbol{Q}$. Note first that $r_{Q}=\operatorname{rank} \hat{T}(\boldsymbol{Q})=\operatorname{rank} \hat{T}^{a}$.

(i) $K$ is real, i.e. $\rho_{1}=1, \rho_{2}=r_{2}=0$.

Since $\hat{T}(R)=\hat{T}, r_{\infty}=\operatorname{dim} T$. Therefore,

$$
r(T)=\operatorname{dim} T-\operatorname{rank} \hat{T}^{G}=r_{\infty}-r_{Q} .
$$

(ii) $K$ is imaginary, i.e. $\rho_{1}=0, \rho_{2}=1, r_{2}=0$.

Since $\hat{T}(R)=\hat{T}^{H}, r(T)=\operatorname{rank} \hat{T}^{H}-\operatorname{rank} \hat{T}^{G}=r_{\infty}-r_{Q}$.

(2.5) Remark. Definition 1.3 and Definition 2.1 are independent of the choice of a splitting field.

Proof. Since the compositum of splitting fields of $T$ is again a splitting field of $T$, it suffices to prove the following:

Let $E$ be an another splitting field of $T$ containing $K$ with galois group $L$, then

$$
\operatorname{Hom}_{L}\left(\hat{T}, O_{E}^{\times}\right) \approx \operatorname{Hom}_{G}\left(\hat{T}, O_{K}^{\times}\right) .
$$

Key point: Assume $\xi \in \operatorname{Hom}_{L}\left(\hat{T}, O_{E}^{\times}\right)$such that $\xi^{\sigma}=\xi$ for all $\sigma \in L=$ $\operatorname{Gal}(E / k)$. Then $\xi^{\sigma}=\xi$ for all $\sigma \in \operatorname{Gal}(E / K)$. Hence $\xi(\hat{T}) \subset O_{K}^{\times}$.

(2.6) Remark. Let $k$ be a number field and $T=R_{k / Q}\left(G_{m}\right)$, where $R$ is the Weil functor (cf. [9] Chapter 1) 
Let $\mathscr{C}(K / k)$ be the category of tori defined over $k$ split by $K$ and $\hat{\mathscr{C}}(K / k)$ be the dual category of finitely generated $Z$-free $\mathrm{Gal}(K / k)$-modules.

We have the following commutative diagram (cf. [7]):

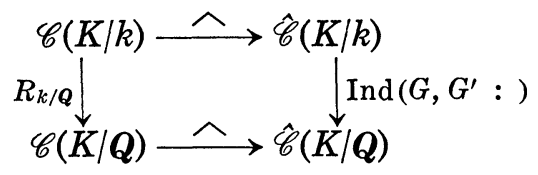

where $G=\operatorname{Gal}(K / Q)$ and $G^{\prime}=\operatorname{Gal}(K / k)$. So

$$
\hat{T}=\widehat{R_{k / \mathbf{Q}\left(G_{m}\right)}}=\hat{G}_{m} \otimes_{z\left[G^{\prime}\right]} Z[G]=Z \otimes_{z\left[G^{\prime}\right]} Z[G]
$$

Therefore,

$$
\begin{aligned}
\operatorname{Hom}_{G}\left(\hat{T}, O_{K}^{\times}\right) & =\operatorname{Hom}_{G}\left(Z \otimes_{z\left[G^{\prime}\right]} Z[G], O_{K}^{\times}\right) \\
& =\left(Z \otimes_{z\left[G^{\prime}\right]} Z[G]\right) \otimes_{\boldsymbol{z}[G]}\left(O_{K}^{\times}\right)^{*} \\
& =Z \otimes_{\boldsymbol{z}\left[G^{\prime}\right]}\left(Z[G] \otimes_{\boldsymbol{z}[G]}\left(O_{K}^{\times}\right)^{*}\right) \\
& =\boldsymbol{Z} \otimes_{\boldsymbol{z}\left[G^{\prime}\right]}\left(O_{K}^{\times}\right)=\operatorname{Hom}_{G^{\prime}}\left(Z, O_{K}^{\times}\right) \\
& =\left(O_{K}^{\times}\right)^{G^{\prime}}=O_{k}^{\times} .
\end{aligned}
$$

We have the following conclusion.

$$
\text { If } T=R_{k / Q}\left(G_{m}\right) \text {, then } T(Z)=O_{k}^{\times} \text {the group of units of } k \text {. }
$$

Note that similar conclusion also holds true for $p$-adic field case.

\section{REFERENCES}

[1] E. Artin, Über Einheiten relativ galoisscher Zählkörper, Crelle Journal, 167 (1932), 153-156.

[2] C. W. Curtis and I. Reiner, Methods of representation theory with application to finite groups and orders, 1, John Wiley \& Sons Inc., 1981.

[3] M. J. Herbrand, Nouvelle démonstration et généralisation d'un théoreme de Minkowski, Comptes rendus, 191 (1930), 1282-1285.

[ 4 ] — Sur les unités d'un corps algébrique, Comptes rendus, 192 (1931), 24-27.

[ 5 ] R. L. Long, Algebraic number theory, Marcel Dekker Inc., 1977, pp. 57-65.

[6] T. Ono, On some arithmetic properties of linear algebraic groups, Ann. of Math., 70, no. 2 (1959), 266-290.

[ 7 ] - Arithmetic of algebraic tori, Ann. of Math., v. 74, no. 1 (1961), 101-119.

[8] - Arithmetic of algebraic groups and its applications, Lecture Notes, Rikkyo 1986.

[ 9 ] A. Weil, Adeles and algebraic groups, Birkhäuser, 1982.

Department of Mathematics

Pohang Institute of Science and Technology

P.O. Box 125 POHANG 790, KOREA 